\title{
Social Isolation, Loneliness, and Mobile Phone Dependence among Adolescents During the COVID-19 Pandemic: Roles of Parent-Child Communication Patterns
}

\author{
Rui Zhen ${ }^{1} \cdot \mathrm{Lu} \mathrm{Li}^{2} \cdot$ Guoqiang $\mathrm{Li}^{1} \cdot \mathrm{Xiao} \mathrm{Zhou}^{3}$
}

Accepted: 27 October 2021

(c) The Author(s), under exclusive licence to Springer Science+Business Media, LLC, part of Springer Nature 2021

\begin{abstract}
During the COVID-19 pandemic, mobile phone dependence (MPD) became a common addictive behavior among adolescents because of social isolation. To understand the underlying mechanisms of the impact of social isolation on MPD, this study examined the mediating role of loneliness and the moderating role of parent-child communication patterns on social isolation and MPD. Six hundred and eighty-three adolescents from a middle school in Huang'gang city, China, were recruited to complete a self-report questionnaire. Data analysis comprised latent profile analysis and structural equation modelling. The results showed that social isolation had a significant and positive indirect effect on MPD via loneliness. In addition, three types of parent-child communication patterns were observed: complex, adaptive, and maladaptive. For adolescents with complex parent-child communication, the indirect relation of social isolation to MPD via loneliness is weaker than that of adolescents with maladaptive parent-child communication. These findings suggested that the indirect effect of social isolation on MPD via loneliness was moderated by parent-child communication patterns. Psychological intervention for MPD should emphasize relieving adolescents' loneliness and improving the quality of their communication with parents during the COVID-19 pandemic.
\end{abstract}

Keywords Social isolation $\cdot$ Loneliness $\cdot$ MPD $\cdot$ Parent-child communication

COVID-19 is an extremely contagious disease with high infectivity and fast transmission, which poses a high risk to all age groups, and has led to widespread public health damage (Dong et al., 2020). To reduce the chances of being infected during the COVID-19 pandemic, the public were asked to stay indoors in quarantine at home for extended periods. Thus, more than 220 million children and adolescents were confined to their homes and

Guoqiang Li

zxzxlgq@126.com

1 Jing Hengyi School of Education, Hangzhou Normal University, Hangzhou 311121, China

2 The No. 1 Middle School of Xiaochi Town in Huangmei County, Huang'gang 435500, China

3 Department of Psychology and Behavioral Sciences, Zhejiang University, Hangzhou 310028, China 
informed that the spring semester would be postponed (Wang et al., 2020). However, to allow children and adolescents to continue studying normally, as they did pre-pandemic, they were asked to use mobile phones or computers to carry out the required online schoolwork, which is considered an effective way to learn. Undoubtedly, as there was a lack of monitoring and evaluation of students who are participating in online courses and/or to the inexperienced and inefficient administration of these courses (Duan et al., 2020), it has facilitated students to show mobile phone dependence (MPD), which refers to overuse of and excessive craving for mobile phone so that individuals are unable to regulate and withdraw (Fu et al., 2020; Zhang et al., 2020). For example, 33.37\% of the adolescents in Dong et al.'s (2020) study were identified as a problematic smartphone user during the COVID19 pandemic. Importantly, overuse of mobile phones or MPD may lead to mental or behavioral problems, including poor academic performance, decreased real-life social interaction, neglect of one's personal life, relationship disorders, and mood dysfunction (Duan et al., 2020; Soni et al., 2017). Thus, we argue that adolescents' MPD should be a target of psychological intervention during widespread health crises like the COVID-19 pandemic.

An important prerequisite to determining which interventions might be most effective for MPD was to clarify the risk factors for MPD during the COVID-19 pandemic and to understand their underlying mechanisms. While researchers have found that many factors influence MPD (Busch \& McCarthy, 2021), social isolation seems to have been a particularly strong factor during the COVID-19 pandemic. It is an objective state measured via indicators such as living alone, having few or infrequent social contacts and low levels of social activity (Dahlberg, 2021), and underling one end of a spectrum encompassing social network size and social contact frequency (Petersen et al., 2016). Stay-at-home and quarantine orders would lead to induced (Kawabe et al., 2020) and enforced social isolation (Fancourt et al., 2021) on an unprecedented scale. Theoretically, people seek relief way through easily accessible means - their smartphones and internet devices-and over-reliance on such coping mechanisms (Elhai et al., 2020). Given this assumption, individuals can turn to the Internet (Chen \& Schulz, 2016; Siste et al., 2020) via their mobile phone or computer to avoid isolation and to meet their social interaction needs in real context, which may increase their risk of MPD. Thus, social isolation may be a risk factor for problematic Internet and smartphone use during widespread forced quarantines such as those imposed during the COVID-19 pandemic (Alheneidi et al., 2021; King et al., 2020).

Another risk factor for MPD may be loneliness. Different from the objective nature of social isolation, loneliness is a subjective negative feeling resulting from a perceived discrepancy between individuals' desired and achieved social connectedness (Perlman \& Peplau, 1981). It has been considered a common psychological reaction during the COVID-19 pandemic (Loades et al., 2020) owing to the implementation of social distancing, lockdown, and quarantine measures (Bu et al., 2020; Dahlberg, 2021; Li \& Wang, 2020). Davis's (2001) cognitive-behavioral model of problematic Internet use provides a possible explanation: individuals who suffer from loneliness are more likely to have distorted cognitions about the self and the world and to develop a strong aversion to and become less satisfied with the physical world (Zhen et al., 2019). In such cases, they may rely on mobile phones to access the world virtually, which may temporarily relieve their loneliness (Kim, 2017); however, this approach may also increase their risk of MPD (Zhen et al., 2019). Indeed, loneliness has been associated with increased MPD (Darcin et al., 2016; Öztunç, 2013).

While a series of quarantine measures may lead to both social isolation and loneliness, thereby causing MPD, social isolation and loneliness are distinct constructs. Moreover, social isolation shows a certain relation to loneliness (Petersen et al., 2016), 
whereby social isolation limits an individual's ability to interact with or in their available social networks, which, in turn, can lead to loneliness (Savikko et al., 2005). Indeed, social isolation is positively correlated with loneliness (Cacioppo et al., 2010). Thus, we proposed that social isolation might have an indirect effect on MPD via loneliness.

As Tang et al. (2021) note, adolescents' parents typically have interacted the most with them during recent home quarantines, and thus, maintaining positive communication with their parents may serve as a protective factor for adolescents' mental health when confronted with social isolation. Furthermore, the effect of social isolation on loneliness and MPD may vary with individual differences in parent-child communication patterns; that is, the role of parent-child communication in adolescents' development is mainly a function of its patterns (Fang et al., 2004; Fitzpatrick \& Ritchie, 1993). Barnes and Olson's (1985) circumplex model suggests that parent-child communication can be viewed as a continuum from open to problematic communication. In this model, open parent-child communication is characterized by the positive disclosure of information between parent and child (Barnes \& Olson, 1985; Metcalfe et al., 2008; Zhou et al., 2020); furthermore, parents are responsive to and affectively involved with their adolescents, who then perceive more positive parental attitudes and behaviors (Fang \& Fang, 2003). Thus, open parent-child communication may help adolescents in home quarantine or social isolation during the pandemic perceive more support and meet their relationship needs, thus relieving their sense of loneliness and reducing their use of mobile phones for engaging in interpersonal interactions. In contrast, problematic communication is characterized by restrained and cautious communication between parents and their children, even to the extent of avoidance in some cases (Fang \& Fang, 2003). Such communication patterns would clearly fall short of meeting adolescents' relationship needs in socially isolated situations such as those experienced during the COVID19 pandemic. Thus, socially isolated adolescents are more likely to be lonely and thus use their mobile phones to satisfy their relationship needs, which in turn increases their risk for MPD.

While open or problematic parent-child communication may exert distinct effects on adolescents' mental and behavioral health, few families show absolute open or problematic parent-child communication in real contexts, and separating one from the other to examine the role of parent-child communication was deemed unrealistic. In fact, each individual may simultaneously experience the two types of parent-child communication in his or her family life, and open and problematic communication will show different combination patterns in different families. Therefore, clarifying the combination patterns can promote a more comprehensive understanding of the heterogeneous characteristics of parent-child communication among adolescent population. Furthermore, because of the widespread implementation of social distancing, lockdowns, and quarantine measures, parent-child communication, social isolation, and loneliness are popular issues that people have been discussing since the COVID-19 pandemic began (Fancourt et al., 2021; Loades et al., 2020; Tang et al., 2021); however, whether these variables have a combined effect on MPD remains unclear. To fill these gaps, this study would examine the moderating role of parent-child communication patterns in the indirecteffect model of the influence of social isolation on MPD via loneliness. Drawing on previous theories and studies, we proposed that different combination patterns of open and problematic parent-child communication would exist in adolescents during the pandemic, and such patterns would moderate the relations between social isolation and MPD via loneliness as a mediator (see Fig. 1). 


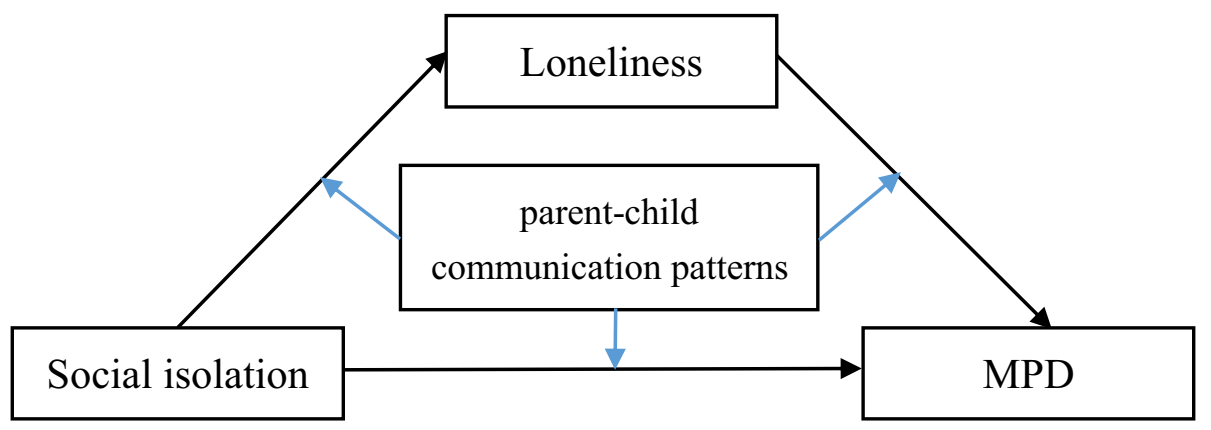

Fig. 1 Hypothesized moderated mediation model

\section{Methods}

\section{Participants and Procedures}

About half a year after the outbreak of COVID-19 pandemic in China, we recruited adolescents from a middle school in Huang'gang city to participate in this investigation during July 16 23, 2020. As Huang'gang is adjacent to Wuhan, it had been severely threatened by the COVID-19 pandemic, and the confirmed COVID-19 cases in Huang'gang city ranked third among a total of 17 cities and districts in Hubei Province. In addition, there were great differences in the pandemic situation in difference places, so that students in some areas had been returned to schools whereas students in other areas might be in strict home quarantine. Therefore, we adopted convenient sampling method to recruit those participants who resumed classes and were willing to participate in this survey. In total, 683 students from a middle school in Huang'gang city were enrolled in this study. Of them, 341 (49.9\%) were male, 301 (44.1\%) were female, and $41(6.0 \%)$ did not report sex. The mean age was 16.06 years (standard deviation 0.56 years; range $15-18$ years).

This study was approved by the Research Ethics Committee of the Department of Psychological and Behavioral Sciences, Zhejiang University. All students in the selected students attended school on the assessment date, and all agreed to participate in the investigation and complete self-report questionnaires. Before the survey, participants were informed of the research purpose and the voluntary nature of participation. Besides, we declared to them that the obtained data would be strictly confidential and would only be used for scientific research. Written informed consent was obtained from all students and their guardians. Assessments were conducted under the supervision of trained psychology postgraduate students.

\section{Measures}

\section{Social Isolation}

Zhen et al., (2021, in press) social isolation questionnaire was used to assess adolescents' social isolation during the COVID-19 pandemic. The questionnaire was developed based on the core conception of social isolation, manifested by how often individuals 
communicate with important others (e. g., family members, friends) in their social network (Taylor et al., 2016). This questionnaire comprised four items (e.g., the frequency for communicating with friends during the COVID-19 pandemic), each rated on a three-point scale: $0=$ "always," $1=$ "sometimes," and $2=$ "never." A higher summed score indicated greater social isolation.

\section{Loneliness}

A state loneliness scale (Gerson \& Perlman, 1979) was used to assess adolescents' loneliness during the COVID-19 pandemic. It comprised 12 items (e.g., lack of friends), each rated on a five-point Likert scale ( $1=$ "completely disagree" to $5=$ "completely agree"). In this study, this scale showed good reliability (Cronbach's $\alpha=0.84$ ).

MPD

A seven-item scale developed by Seo et al. (2016) was used to assess adolescents' MPD. Responses on each item were rated on a four-point Likert scale (1= "strongly no" to $4=$ "strongly yes"); a higher aggregated score indicated greater MPD. The scale showed good reliability in previous studies (Fu et al., 2020; Zhen et al., 2020) and in the current study (Cronbach's $\alpha=0.85$ ).

\section{Parent-child Communication}

The Chinese version of a parent-child communication scale was used to assess parent-child communication (An, 2004). This scale comprised 20 items, divided into two dimensions: openness and problems in parent-child communication. Items were rated on a five-point Likert scale ( $1=$ "completely disagree" to $5=$ "completely agree"). This scale has previously shown good reliability and validity with adolescents (Zhou et al., 2020) and also showed good reliability in the present study for both open parent-child communication (Cronbach's alpha $=0.91$ ) and problematic parent-child communication (Cronbach's alpha $=0.79$ ).

\section{Data Analysis Procedures}

This study had two aims: assessing the latent combination patterns of open and problematic communication and examining the moderating role of such patterns in the relations among social isolation, loneliness, and MPD. In view of the first aim, we used Mplus 7.0 to conduct latent profile analysis based on adolescents' open and problematic parent-child communication. To determine the optimal number of latent classes, 1- to 5-class solutions were evaluated and compared on the basis of fit statistics, interpretability, and theoretical considerations. A good model fit was indicated by lower Bayesian information criterion, adjusted Bayesian information criterion, and Akaike information criterion, higher entropy, a significant Lo-Mendell-Rubin likelihood ratio test (LMR-LRT), and a significant adjusted LMR-LRT (ALMR-LRT). After determining the optimal class, the most likely class membership variables were then exported in the set of parent-child communication patterns data to SPSS21.0, and the following analysis was conducted.

In view of the second aim, several analysis procedures were performed. First, we built a direct-effect model, with social isolation predicting MPD. Second, we built an 
indirect-effect model in Mplus 7.0, with loneliness mediating the relationship between social isolation and MPD. To evaluate model fits, we used chi-square values, the comparative fit index (CFI), the Tucker-Lewis index (TLI), the root mean square error of approximation (RMSEA), and the standardized root mean residual (SRMR). The cutoffs for accepting a model were equal to or greater than 0.90 for CFI and TLI and equal to or less than 0.08 for the RMSEA and SRMR. Next, a multiple-group comparison model in Amos 20.0 was built to examine the moderating role of parent-child communication patterns on the indirect-effect model. Based on our hypothesis that parent-child communication patterns could play a moderating role in the direct effect of social isolation on loneliness and MPD, we then constrained the predictive paths from social isolation to loneliness and MPD in distinct parent-child communication patterns model to be equal. Next, following the suggestions of Farrell (1994), we compared the measurement parameters between the two groups, as there were no structural parameters. The difference in the CFI between the unconstrained and constrained models was more than 0.01 which informs a significant difference of two models (Cheung \& Rensvold, 2002), suggesting a significant moderating effect.

\section{Results}

\section{Descriptive Statistics and Correlation Analysis}

Table 1 presents the descriptive statistics and correlations among the main variables. We observed a significant and positive correlation between social isolation, loneliness, MPD, and problematic parent-child communication. Open parent-child communication was significantly and negatively related to the other variables.

\section{Assessment of Parent-Child Communication Patterns}

Table 2 shows the latent class solution for both open and problematic parent-child communications among participating adolescents. We found that the value of entropy exceeded 0.70 for the 3- to 6-class solutions, suggesting that the 3- to 6-class solutions had better between-group distinctions than other solutions. Moreover, the findings indicated that the LMR-LRT and ALMR-LRT values were significant for the 2-, 3-, and 5-class solutions but not for the other solutions. The prevailing standard is that when the LMR-LRT and

Table 1 Descriptive statistics and correlation analysis among main variables

\begin{tabular}{|c|c|c|c|c|c|}
\hline Variables & $\mathrm{M}(\mathrm{SD})$ & 1 & 2 & 3 & 4 \\
\hline 1. Social isolation & $3.97(1.48)$ & 1.00 & & & \\
\hline 2. Loneliness & $31.47(7.81)$ & $0.38^{* * *}$ & 1.00 & & \\
\hline 3. MPD & $17.94(4.69)$ & $0.12^{* *}$ & $0.28^{* * *}$ & 1.00 & \\
\hline 4. Open communication & $18.35(8.68)$ & $-0.40^{* * *}$ & $-0.44^{* * *}$ & $-0.14^{* * *}$ & 1.00 \\
\hline 5. Problematic communication & $20.14(7.24)$ & $0.32^{* * *}$ & $0.42^{* * *}$ & $0.30^{* * *}$ & $-0.56^{* * *}$ \\
\hline
\end{tabular}

Open communication $=$ open parent-child communication; Problematic communication $=$ problematic parent-child communication

${ }^{* * *} p<0.001,{ }^{* *} p<0.01$ 
Table 2 Goodness of fit indices for distinct solutions

\begin{tabular}{lllllll}
\hline Class & AIC & BIC & adjBIC & Entropy & LMR-LRT & ALMR-LRT \\
\hline 1-Class & 9274.701 & 9292.789 & 9280.089 & - & - & - \\
2-Classes & 9127.950 & 9159.604 & 9137.379 & 0.612 & $152.751^{* *}$ & $145.324^{* *}$ \\
3-Classes & $\mathbf{9 0 1 5 . 1 4 6}$ & $\mathbf{9 0 6 0 . 3 6 7}$ & $\mathbf{9 0 2 8 . 6 1 5}$ & $\mathbf{0 . 7 3 6}$ & $\mathbf{1 1 8 . 8 0 4}$ *** $^{* * *}$ & $\mathbf{1 1 3 . 0 2 7}^{* * *}$ \\
4-Classes & 8999.893 & 9058.680 & 9017.404 & 0.791 & 21.253 & 20.219 \\
5-Classes & 8980.268 & 9052.622 & 9001.820 & 0.771 & $25.625^{* *}$ & $24.379^{* *}$ \\
6-Classes & 8976.306 & 9062.226 & 9001.899 & 0.775 & 9.962 & 9.477 \\
\hline
\end{tabular}

${ }^{* * *} p<0.001,{ }^{* *} p<0.01$. The 3 -Classes in bold font indicates the optimal solution.

ALMR-LRT values are significant in many classes of solutions, the solution with the largest number of classes should be accepted (Rosellini, Coffey, Tracy, \& Galea, 2014). In light of these criteria, 3- and 5-class were considered in this study. However, we found that one of the classes in the 5-class solution only had eight participants (1.2\%), suggesting that the 5 -class solution was unstable and should thus be excluded as an optimal solution. We then selected the 3-class salutation as optimal for this study.

Table 3 shows the unstandardized scores for open and problematic parent-child communication in three types of patterns, as well as the results of the analyses of variance and post hoc pairwise comparisons. We found that between-class differences in open and problematic parent-child communications were significant. As Table 3 shows, for the first group, the level of open parent-child communication was slightly higher than that of its population mean (18.35), while problematic parent-child communication was slightly less than that of the population mean (20.14). This suggested that these adolescents had both medium-levels of open and problematic parent-child communication and thus we referred to this group as the complex communication group $(n=467,68.7 \%)$. The second group, which we named the adaptive communication group $(n=78,11.5 \%)$, had high levels of open parent-child communication and low levels of problematic parent-child communication. The third group, called the maladaptive communication group $(n=135,19.9 \%)$, had high levels of problematic parent-child communication and low levels of open parent-child communication.

\section{Examination of the Moderated Mediating Model}

A direct-effect model in which social isolation predicted MPD fits the data completely, $\chi^{2}(0)=0.00, \mathrm{CFI}=1.00, \mathrm{IFI}=1.00$, RMSEA $(90 \% \mathrm{CI})=0.00(0.00-0.00)$. Social isolation was significantly positively related to MPD $(\beta=0.12, p<0.01)$. Next, we put the loneliness variable between social isolation and MPD and built the indirect-effect model (Fig. 2). This model fits the data completely, $\chi^{2}(0)=0.00, \mathrm{CFI}=1.00, \mathrm{IFI}=1.00$, RMSEA $(90 \%$ $\mathrm{CI})=0.00(0.00-0.00)$. Using path analysis, we found that the direct path from social isolation to MPD was non-significant, but social isolation had an indirect positive effect on MPD via loneliness. These results suggested that loneliness played a partial mediating role between social isolation and MPD.

Next, based on the mediation effect model in Fig. 2, multiple group analysis was conducted to assess whether the mediating effect of loneliness between social isolation and MPD differed between the complex $(n=467)$, adaptive $(n=78)$, and maladaptive $(n=135)$ 


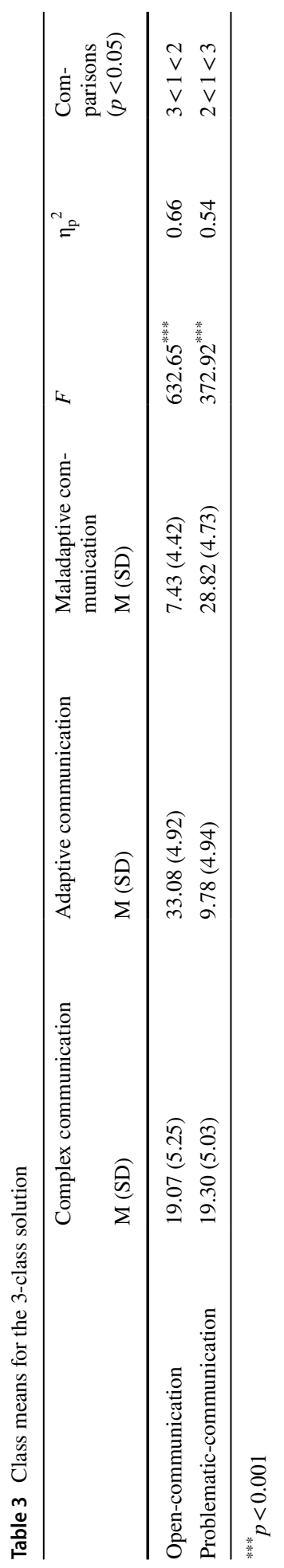




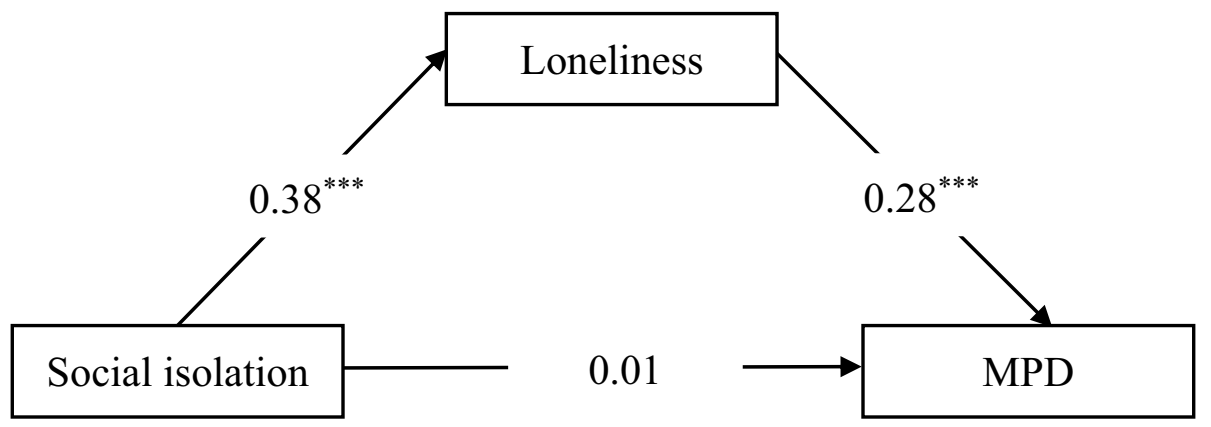

Fig. 2 Mediation effect model. ${ }^{* * *} p<0.001$

communication groups. Following the suggestions of Farrell (1994), we compared the measurement parameters between these three groups, as there were no structural parameters. In terms of measurement parameters, all the paths were constrained to be equal. The results showed that the measurement model had good fit indices: $\chi^{2}(6)=10.29$, $\mathrm{CFI}=0.95, \mathrm{IFI}=0.95$, RMSEA $(90 \% \mathrm{CI})=0.03(0.00-0.07)$. A significant difference was found between the constrained model and the non-constrained model: $\Delta \mathrm{CFI}=0.051$ and $\Delta$ IFI $=0.049$. The results indicated that the mediation model was moderated by parent-child communication patterns. The critical ratios for the differences between parameters (Table 4) indicated that the predictive path from social isolation to loneliness differed between complex communication and maladaptive communication: the positive relation between social isolation and loneliness in the maladaptive communication group $(\beta=0.37$, $p<0.05)$ was stronger than that in the complex communication group $(\beta=0.22, p<0.05)$. All the other predictive paths had non-significant differences between the two or three communication patterns.

\section{Discussion}

During the COVID-19 pandemic, the implementation of home quarantines and social distancing measures has highlighted the need to investigate and facilitate the assessment of the underlying mechanisms of MPD from the perspective of social isolation, loneliness, and parent-child communication, which have been separately discussed by researchers as common issues since the beginning of the pandemic (Fancourt et al., 2021; Faustino et al., 2021; Loades et al., 2020; Tang et al., 2021). This is the first attempt to clarify the

Table 4 Critical ratios for differences between parameters in multiple-group analysis

\begin{tabular}{llll}
\hline Predictive paths & $\begin{array}{l}\text { Complex } v s \text { maladaptive } \\
\text { communication }\end{array}$ & $\begin{array}{l}\text { Adaptive } v s \text { maladaptive } \\
\text { communication }\end{array}$ & $\begin{array}{l}\text { Complex vs adap- } \\
\text { tive communica- } \\
\text { tion }\end{array}$ \\
\hline Social isolation $\rightarrow$ MPD & $C R=-0.20, p>0.05$ & $C R=-1.00, p>0.05$ & $C R=1.10, p>0.05$ \\
Social isolation $\rightarrow$ Loneliness & $C R=2.22, p<0.05$ & $C R=0.27, p>0.05$ & $C R=1.49, p>0.05$ \\
Loneliness $\rightarrow$ MPD & $C R=-0.50, p>0.05$ & $C R=-1.22, p>0.05$ & $C R=0.97, p>0.05$ \\
\hline
\end{tabular}


combination patterns of open-communication and problematic-communication in an adolescent sample during the COVID-19 pandemic. More importantly, this research firstly revealed the interactive influencing mechanism among such patterns, social isolation, loneliness, and adolescents' MPD.

In specific, we found that social isolation had a direct positive relation with MPD, which is consistent with previous findings (Al-Kandari \& Al-Sejari, 2020). In fact, social isolation is considered a social alienation dimension (Al-Kandari \& Al-Qashan, 2001), wherein individuals have difficulty in developing friendships in real life (Al-Kandari \& Al-Sejari, 2020). They may then resort to mobile phone for online communication and social interaction, as suggested by the social compensation hypothesis (McKenna et al., 2002). Previous research found that using smartphone apps of communication and social networking did reduce individuals' social isolation and improve their personal lives (Cho, 2015). Hence, social isolation directly impelled adolescents during the pandemic to a high possibility of MPD.

Furthermore, the research found that adolescents' subjective lonely state completely mediated the relation between objective social isolation and their MPD. This suggested that the ultimate cause for these adolescents to depend on mobile phones lied in their internal loneliness. Social isolation in the pandemic may initially separate adolescents in objective situations and limited their interpersonal interaction (Lewis, 2020). However, this made it difficult to satisfy their basic psychological needs of relatedness and sense of belongingness, which increased the discrepancy between desired and achieved social connectedness (Perlman \& Peplau, 1981), eliciting adolescents' loneliness (Mellor et al., 2008). Especially in the context of the stressful COVID-19 event, the daily social network of these adolescents was suddenly cut off, and their perceived loneliness boosted in a short time, so they are more likely to make up for the sharp deletion of interpersonal intimacy and social support in real life through various mobile network means (Caplan, 2007; Ceyhan \& Ceyhan, 2008; McKenna et al., 2002). As a result, the lonely feelings generated from social isolation urged adolescents to experience increased MPD.

Each of the three distinct parent-child communication patterns that derived from the latent profile analysis-complex, adaptive, and maladaptive-contained both open and problematic communication components, suggesting that parent-child communication during the COVID-19 pandemic was likely neither absolutely open nor absolutely problematic, and was heterogeneous among adolescents. Interestingly, the complex communication group had the largest population $(n=467,68.7 \%)$, followed by the maladaptive communication group $(n=135,19.9 \%)$ and finally adaptive communication group $(n=78,11.5 \%)$. The complex group is characterized by equivalent medium levels of open and problematic parent-child communication. On the one hand, the measures of home quarantine made family members to stay together every day, which had created opportunities for parents and children to confide in each other and showed an open communication style. But on the other hand, quarantine at home aroused the conflicts between parents and children when academic supervision, housework sharing, and other household chores were not well handled (Chen et al., 2021). For example, a survey on 12,711 parents showed that compared with last year, $31.4 \%$ of them reported an increase in the frequency of parent-child conflicts during the COVID-19 pandemic in China (Chen et al., 2021). Such conflicts may trigger problematic parent-child communication style. Hence, the major adolescents displayed the coexisting pattern of open and problematic communication during the pandemic.

More importantly, parent-child communication patterns moderated the path from social isolation to loneliness. In specific, the positive relation between social isolation and loneliness in the maladaptive communication group was stronger than that in the 
complex communication group. Adolescents with the maladaptive communication pattern avoided discussing or communicating with their parents; thus, these socially isolated ones could not meet their relatedness needs and were likely to involve in negative cognition, and finally they perceived a deep sense of loneliness. Differently, adolescents with the complex communication pattern had a medium level of open communication with their parents, in which children to a certain degree felt supported by parents and perceived positive parental attitudes (Fang \& Fang, 2003), so their loneliness brought by social isolation was weaker. What is unexpected, there was no significant difference in the relation of social isolation to loneliness between the maladaptive and the adaptive communication group. Although adaptive communication pattern had a high level of open communication, with which parents and children had more disclosure and support for each other, sharing worries and concerns may also cause family members to experience symptoms of psychological distress (Teyber, 1983). This may be due to the contagious effect of emotions (Hatfield et al., 1993), which endows adolescents to perceive their parents' negative emotions under the pandemic and then show similar emotions. Hence, isolated adolescents with adaptive communication pattern did not perceive reduced loneliness. Such finding suggested that an appropriate level of communication between parents and children was optimal when encountering in stressful events such as the COVID-19 pandemic.

Several limitations in this study should be noted. First, this is a cross-sectional research design, so all the causal language is on the basis of theoretical assumptions and no causal conclusions can be drawn. Second, interpersonal interactions are important for adolescents, but we only took parent-child communication into account and neglected other types of interpersonal communication (e.g., with peers). Third, as measures of quarantine and social distancing are different in distinct developmental phases of the pandemic, adolescents' experiences of social isolation and loneliness may vary in different phases and different pandemic severity (Murayama et al., 2021). Therefore, these factors need to be considered when interpreting the results of this study. Finally, this study only included some adolescents from one city in Hubei Province, China; the selective-bias sampling reminds that any generalizations of the findings to population of other age groups in other areas should be made with caution.

Notwithstanding these limitations, this is the first study to examine the combined role of social isolation, loneliness, and parent-child communication in MPD during the COVID-19 pandemic. The positive effects of social isolation on MPD via only loneliness reflect that MPD's core factor was the subjective psychological state elicited by objective isolation, home quarantine, and social distancing. Moreover, while parent-child communication patterns moderated the indirect effect of social isolation on MPD via loneliness, those patterns did not change the predictive valence of social isolation's influence on MPD via loneliness. This finding indicates that when adolescents are socially isolated, they may nevertheless be lonely and thus at risk for MPD, irrespective of the type of communication pattern they have with their parents. From a clinical perspective, psychological intervention for MPD should be emphasized to relieve adolescents' psychological problems, particularly loneliness during forced isolation such as that experienced during the COVID-19 pandemic. Additionally, once the pandemic has diminished, an effective strategy to relieve adolescents' MPD may be to increase adolescents' social communication, in addition to parents. 
Funding This work was supported by the National Youth Project for National Social Sciences of China (Education) [Grant No. CHA200259].

\section{Declarations}

Ethical Approval This study was approved by the Research Ethics Committee of the Department of Psychological and Behavioral Sciences, Zhejiang University. Informed consent was obtained from each participant included in our investigation.

Conflict of Interest The authors declare no competing interests.

\section{References}

Al-Kandari, Y., \& Al-Qashan, H. (2001). The relationship between the use of the internet and the social isolation among students at Kuwait University. Journal for Humanities and Social Science, 17, 1-45.

Al-Kandari, Y. Y., \& Al-Sejari, M. M. (2020). Social isolation, social support and their relationship with smartphone addiction. Information Communication \& Society. https://doi.org/10.1080/1369118x.2020. 1749698

Alheneidi, H., AlSumait, L., AlSumait, D., \& Smith, A. P. (2021). Loneliness and problematic internet use during COVID-19 lock-down. Behavioral Sciences, 11(5), 1-11. https://doi.org/10.3390/bs11010005

An, B. (2004). Parenting style, parent-adolescent communication and their effect on adolescents' social adjustment (Unpublished master's dissertation, Shannxi University)

Barnes, H. L., \& Olson, D. H. (1985). Parent-adolescent communication and the circumplex model. Child Development, 56, 438-447.

Brand, M., Wegmann, E., Stark, R., Mueller, A., Woelfling, K., Robbins, T. W., \& Potenza, M. N. (2019). The Interaction of Person-Affect-Cognition-Execution (I-PACE) model for addictive behaviors: Update, generalization to addictive behaviors beyond internet-use disorders, and specification of the process character of addictive behaviors. Neuroscience and Biobehavioral Reviews, 104, 1-10. https:// doi.org/10.1016/j.neubiorev.2019.06.032

Bu, F., Steptoe, A., \& Fancourt, D. (2020). Who is lonely in lockdown? Cross-cohort analyses of predictors of loneliness before and during the COVID-19 pandemic. Public Health, 186, 31-34.

Busch, P. A., \& McCarthy, S. (2021). Antecedents and consequences of problematic smartphone use: A systematic literature review of an emerging research area. Computers in Human Behavior, 114, 106414. https://doi.org/10.1016/j.chb.2020.106414

Cacioppo, J. T., Hawkley, L. C., \& Thisted, R. A. (2010). Perceived social isolation makes me sad: 5-year cross-lagged analyses of loneliness and depressive symptomatology in the Chicago health, aging, and social relations study. Psychology and Aging, 25, 453-463. https://doi.org/10.1037/a0017216

Caplan, S. E. (2007). Relations among loneliness, social anxiety, and problematic internet use. CyberPsychology \& Behavior, 10(2), 234-242. https://doi.org/10.1089/cpb.2006.9963

Ceyhan, A. A., \& Ceyhan, E. (2008). Loneliness, depression, and computer self-efficacy as predictors of problematic internet use. CyberPsychology \& Behavior, 11(6), 699-701. https://doi.org/10.1089/cpb. 2007.0255

Chen, Y., Cui, L., Liu, L., \& Lu, F. (2021). Parent-child conflict among primary and middle school students during the COVID-19 epidemic and its countermeasures. Chinese Journal of School Health, 42(5), 719-722. https://doi.org/10.16835/j.cnki.1000-9817.2021.05.019

Chen, Y.-R. R., \& Schulz, P. J. (2016). The effect of information communication technology interventions on reducing social isolation in the elderly: A systematic review. Journal of Medical Internet Research, 18(1), e18. https://doi.org/10.2196/jmir.4596

Cheung, G. W., \& Rensvold, R. B. (2002). Evaluating goodness-of-fit indexes for testing measurement invariance. Structural Equation Modeling A Multidisciplinary Journal, 9(2), 233-255.

Cho, J. (2015). Roles of smartphone app use in improving social capital and reducing social isolation. Cyberpsychology Behavior \& Social Networking, 18(6), 350-355. https://doi.org/10.1089/cyber.2014. 0657

Dahlberg, L. (2021). Loneliness during the COVID-19 pandemic. Aging \& Mental Health. https://doi.org/ $10.1080 / 13607863.2021 .1875195$ 
Darcin, A. E., Kose, S., Noyan, C. O., Nurmedov, S., Yilmaz, O., \& Dilbaz, N. (2016). Smartphone addiction and its relationship with social anxiety and loneliness. Behaviour \& Information Technology, 35, 520-525. https://doi.org/10.1080/0144929x.2016.1158319

Davis, R. A. (2001). A cognitive-behavioral model of pathological Internet use. Computers in Human Behavior, 17, 187-195. https://doi.org/10.1016/s0747-5632(00)00041-8

Dong, H., Yang, F., Lu, X., \& Hao, W. (2020). Internet addiction and related psychological factors among children and adolescents in China during the Coronavirus Disease 2019 (COVID-19) epidemic. Frontiers in psychiatry, 11, 00751. https://doi.org/10.3389/fpsyt.2020.00751

Duan, L., Shao, X., Wang, Y., Huang, Y., Miao, J., Yang, X., \& Zhu, G. (2020). An investigation of mental health status of children and adolescents in china during the outbreak of COVID-19. Journal of Affective Disorders, 275, 112-118. https://doi.org/10.1016/j.jad.2020.06.029

Elhai, J. D., Yang, H., McKay, D., \& Asmundson, G. J. G. (2020). COVID-19 anxiety symptoms associated with problematic smartphone use severity in Chinese adults. Journal of Affective Disorders, 274, 576-582. https://doi.org/10.1016/j.jad.2020.05.080

Fancourt, D., Steptoe, A., \& Bu, F. (2021). Trajectories of anxiety and depressive symptoms during enforced isolation due to COVID-19 in England: A longitudinal observational study. The Lancet Psychiatry, 8, 141-149. https://doi.org/10.1016/s2215-0366(20)30482-X

Fang, C., \& Fang, X. (2003). Review: Parent-adolescent communication research. Advances in Psychological Science, 11, 65-72.

Fang, X., Lin, D., Sun, L., \& Fang, C. (2004). Parent-Adolescent Communication and Adolescents' Social Adjustment. Psychological Development and Education, 20, 18-22.

Farrell, A. D. (1994). Structural equation modeling with longitudinal data: Strategies for examining group differences and reciprocal relationships. Journal of Consulting and Clinical Psychology, 62, 477-487. https://doi.org/10.1037/0022-006x.62.3.477

Faustino, B., Vasco, A. B., Delgado, J., Farinha-Fernandes, A., \& Guerreiro, J. C. (2021). Exploring the impacts of COVID-19 related social distancing on loneliness, psychological needs and symptomatology. Research in Psychotherapy Psychopathology Process and Outcome, 23, 222-230.

Fitzpatrick, M. A., \& Ritchie, L. D. (1993). Communication theory and the family. In P. Boss, W. J. Doherty, R. LaRossa, W. R. Schumm, \& S. K. Steinmetz (Eds.), Sourcebook of Family Theories and Methods: A Contextual Approach (pp. 565-589). Springer, US.

Fu, X. C., Liu, J. X., Liu, R. D., Ding, Y., Hong, W., \& Jiang, S. Y. (2020). The impact of parental active mediation on adolescent mobile phone dependency: A moderated mediation model. Computers in Human Behaviors, 107, 106280. https://doi.org/10.1016/j.chb.2020.106280

Gerson, A. C., \& Perlman, D. (1979). Loneliness and expressive communication. Journal of Abnormal Psychology, 88, 258-261.

Hatfield, E., Cacioppo, J. T., \& Rapson, R. L. (1993). Emotional contagion. Current Directions in Psychological Science, 2, 96-100. https://doi.org/10.1111/1467-8721.ep10770953

Kawabe, K., Hosokawa, R., Nakachi, K., Yoshino, A., Horiuchi, F., \& Ueno, S.-I. (2020). Excessive and problematic internet use during the Coronavirus Disease 2019 school closure: Comparison between Japanese youth with and without autism spectrum disorder. Frontiers in Public Health, 8, 609347. https://doi.org/10.3389/fpubh.2020.609347

Kim, J.-H. (2017). Smartphone-mediated communication vs. face-to-face interaction: Two routes to social support and problematic use of smartphone. Computers in Human Behavior, 67, 282-291. https://doi. org/10.1016/j.chb.2016.11.004

King, D. L., Delfabbro, P. H., Billieux, J., \& Potenza, M. N. (2020). Problematic online gaming and the COVID-19 pandemic. Journal of Behavioral Addictions, 9, 184-186. https://doi.org/10.1556/2006. 2020.00016

Lewis, K. (2020). COVID-19: Preliminary data on the impact of social distancing on loneliness and mental health. Journal of Psychiatric Practice, 26, 400-404.

Li, L. Z., \& Wang, S. (2020). Prevalence and predictors of general psychiatric disorders and loneliness during COVID-19 in the United Kingdom. Psychiatry Research, 291, 113267. https://doi.org/10.1016/j. psychres.2020.113267

Loades, M. E., Chatburn, E., Higson-Sweeney, N., Reynolds, S., Shafran, R., Brigden, A., . . Crawley, E. (2020). Rapid systematic review: The impact of social isolation and loneliness on the mental health of children and adolescents in the context of COVID-19. Journal of the American Academy of Child and Adolescent Psychiatry, 59, 1218-1239. https://doi.org/10.1016/j.jaac.2020.05.009

McKenna, K. Y. A., Green, A. S., \& Gleason, M. E. J. (2002). Relationship formation on the internet: What's the big attraction? Journal of Social Issues, 58, 9-31. https://doi.org/10.1111/1540-4560.00246 
Mellor, D., Stokes, M., Firth, L., Hayashi, Y., \& Cummins, R. (2008). Need for belonging, relationship satisfaction, loneliness, and life satisfaction. Personality and Individual Differences, 45(3), 213-218. https://doi.org/10.1016/j.paid.2008.03.020

Metcalfe, A., Coad, J., Plumridge, G. M., Gill, P., \& Farndon, P. (2008). Family communication between children and their parents about inherited genetic conditions: A meta-synthesis of the research. European Journal of Human Genetics, 16, 1193-1200. https://doi.org/10.1038/ejhg.2008.84

Murayama, H., Okubo, R., \& Tabuchi, T. (2021). Increase in social isolation during the COVID-19 pandemic and its association with mental health: Findings from the JACSIS 2020 study. International Journal of Environmental Research and Public Health, 18(16) , 8238. https://doi.org/10.3390/ijerp h18168238

Öztunç, M. (2013). Analysis of problematic mobile phone use, feelings of shyness and loneliness in accordance with several variables. Procedia - Social and Behavioral Sciences, 106, 456-466. https://doi.org/ 10.1016/j.sbspro.2013.12.051

Perlman, D., \& Peplau, L. A. (1981). Toward a social psychology of loneliness. In S. Duck \& R. Gilmour (Eds.), Personal relationships in disorder (pp. 31-56). Academic Press.

Petersen, J., Kaye, J., Jacobs, P. G., Quinones, A., Dodge, H., Arnold, A., \& Thielke, S. (2016). Longitudinal relationship between loneliness and social isolation in older adults: Results from the cardiovascular health study. Journal of Aging and Health, 28, 775-795. https://doi.org/10.1177/0898264315611664

Rosellini, A. J., Coffey, S. F., Tracy, M., \& Galea, S (2014). A person-centered analysis of posttraumatic stress disorder symptoms following a natural disaster: Predictors of latent class membership. Journal of Anxiety Disorders, 28(1), 16-24. https://doi.org/10.1016/j.janxdis.2013.11.002

Savikko, N., Routasalo, P., Tilvis, R. S., Strandberg, T. E., \& Pitkala, K. H. (2005). Predictors and subjective causes of loneliness in an aged population. Archives of Gerontology and Geriatrics, 41, 223-233. https://doi.org/10.1016/j.archger.2005.03.002

Seo, D. G., Park, Y., Kim, M. K., \& Park, J. (2016). Mobile phone dependency and its impacts on adolescents' social and academic behaviors. Computers in Human Behavior, 63, 282-292. https://doi.org/10. 1016/j.chb.2016.05.026

Siste, K., Hanafi, E., Sen, L. T., Christian, H., Adrian, Siswidiani, L. P., . . S Suwartono, C. (2020). The impact of physical distancing and associated factors towards internet addiction among adults in Indonesia during COVID-19 pandemic: A nationwide web-based study. Frontiers in Psychiatry, 11. https:// doi.org/10.3389/fpsyt.2020.580977

Soni, R., Upadhyay, R., \& Jain, M. (2017). Prevalence of smart phone addiction, sleep quality and associated behaviour problems in adolescents. International Journal of Research in Medical Sciences, 5, 515-519.

Tang, S., Xiang, M., Cheung, T., \& Xiang, Y.-T. (2021). Mental health and its correlates among children and adolescents during COVID-19 school closure: The importance of parent-child discussion. Journal of Affective Disorders, 279, 353-360. https://doi.org/10.1016/j.jad.2020.10.016

Taylor, R. J., Taylor, H. O., \& Chatters, L. M. (2016). Social isolation from extended family members and friends among African Americans: Findings from a national survey. Journal of Family Social Work, 19(5), 443-461.

Teyber, E. (1983). Effects of the parental coalition on adolescent emancipation from the family. Journal of Marital and Family Therapy, 9(3), 305-310. https://doi.org/10.1111/j.1752-0606.1983.tb01515.x

Wang, G., Zhang, Y., Zhao, J., Zhang, J., \& Jiang, F. (2020). Mitigate the effects of home confinement on children during the COVID-19 outbreak. Lancet, 395, 945-947. https://doi.org/10.1016/s01406736(20)30547-X

Zhang, G., Yang, X., Tu, X., Ding, N., \& Lau, J. T. F. (2020). Prospective relationships between mobile phone dependence and mental health status among Chinese undergraduate students with college adjustment as a mediator. Journal of Affective Disorders, 260, 498-505. https://doi.org/10.1016/j.jad. 2019.09.047

Zhen, R., Li, L., \& Zhou, X. (2021). The mediation roles of psychological needs satisfaction and self-disclosure between social isolation and PTSD/PTG among adolescents under the COVID-19 epidemic. Chinese Journal of Clinical Psychology, 29(5), 967-972.

Zhen, R., Li, L., Liu, X., \& Zhou, X. (2020). Negative life events, depression, and mobile phone dependency among left-behind adolescents in rural China: An interpersonal perspective. Children and Youth Services Review, 109, 104688. https://doi.org/10.1016/j.childyouth.2019.104688

Zhen, R., Liu, R. D., Hong, W., \& Zhou, X. (2019). How do interpersonal relationships relieve adolescents' problematic mobile phone use? The roles of loneliness and motivation to use mobile phones. Intertional Journal of Environment Research amd Public Health, 16(13), 2286. https://doi.org/10.3390/ ijerph16132286 
Zhou, X., Zhen, R., \& Wu, X. (2020). Insecure attachment to parents and PTSD among adolescents: The roles of parent-child communication, perceived parental depression, and intrusive rumination. Development and Psychopathology. https://doi.org/10.1017/s0954579420000498

Publisher's Note Springer Nature remains neutral with regard to jurisdictional claims in published maps and institutional affiliations. 